a la plaza de profesor de Botánica del Museo de Historia Natural, regresó a Málaga [...]”. J. L. Carrillo. En: M. Alcobendas (ed.). Málaga. Personajes en su historia: 357-358. Málaga. 1986.

${ }^{9}$ Transcribía el cura de la parroquia de San Juan (Málaga, 10/5/1832) la partida inscrita en el Libro de Matrimonios $\mathrm{n}^{\circ}$ 23, folio 278, fechada el 6/7/1790. Los padres de Pablo Prolongo se desposaron "por palabra de presente que hicieron verdadero y legítimo matrimonio y veló con las ceremonias del ritual romano". El mismo párroco copió la partida del Libro de Bautismos $\mathrm{n}^{\circ} 80$, folio 78 , datada el 30/5/1806, en el que constan los tres nombres con que recibió en el sacramento: Pablo Justo Rafael. "Declaró su padre no haber tenido otro hijo del mismo nombre y aseguró con juramento que nació el día 28 del corriente". ${ }^{10}$ Figuraba en el Real Despacho de nombramiento de capitán graduado de infantería del 17/5/1794: "Por cuanto atendiendo a los servicios de vos el teniente D. Antonio Prolongo, comandante de uno de los jabeques de Melilla, y al particular mérito que últimamente habéis contraído en la aprehensión de un barco contrabandista; he venido en concederos grado de capitán de infantería [...]".

Dirección del autor: Centro Oceanográfico de Málaga. Instituto Español de Oceanografía. Muelle Pesquero s/n. Apdo. 285. 29.640 Fuengirola (Málaga). Dirección de contacto:jprubin@ma.ieo.es.

\title{
45. UN ARTÍCULO DE 1880 SOBRE EL 'JARABE DE PINSAPO' ELABORADO EN LA FARMACIA DEL MALAGUEÑO PABLO PROLONGO GARCÍA (1806-1885)
}

\author{
Manuel GARRIDO y Juan PÉREZ-RUBÍN
}

A. González Bueno analizó concienzudamente el primer viaje botánico del ginebrino Pierre-Edmond Boissier (1810-1885) por el sur de España en 1837, que incluyó una excursión en compañía de los boticarios de Málaga Félix Haenseler (1767-1841) y Pablo Prolongo (1806-1885) en busca del pinsapo, famoso 'pino' de las montañas de Estepona: "una mañana de finales de septiembre, prácticamente ultimada ya su estancia andaluza, inicia [Boissier] una expedición a la Sierra de las Nieves para estudiar sus pinsapos" ${ }^{\text {"1. }}$. Dos décadas antes que Boissier, el español Simón de Rojas Clemente (1777-1827) ya había publicado una descripción del pinsapo en la que demuestra conocer sus piñas (1818) y concreta su distribución geográfica: "abunda espontáneo en la sierra del Pinar, en la de Tolox y la de los Reales sobre Estepona, a la altura de unas 1.900 hasta 2.400 varas sobre el nivel del mar; siendo su zona favorita la subalpina $[\ldots]^{\prime 2}$.

En el artículo del propio Prolongo de 1880, que transcribimos aquí, comentaba un detalle muy importante que creemos olvidado actualmente: que él fue encargado por ambos compañeros de excursión botánica: "para describir los órganos florales de dicha planta, en la primavera próxima [1838], con cuyos 
datos obtenidos por mí quedó clasificado Abies insapo (de Boissier)". El botánico suizo había reconocido ese año que era una tarea pendiente para sus colegas de Málaga: “Comme il m’a été impossible d'observer les organes mâles et femelles à l'époque de la floraison, je n'en parlerai pas. J'espère remplir plus tard cette lacune, grâce à l'obligeance de mes amis de Málaga”. E. Boissier acabó dedicando al botánico y boticario malagueño algunas de las especies nuevas que encontraron, tales como Diplotaxis prolongi [prolongoi] y Thlaspi prolongoi, además del género Prolongoa perteneciente a las Compuestas. Idéntica y valiosa ayuda prestó Prolongo a los ilustres naturalistas Kelaerd y Wilkomm, que en 1845 vinieron a nuestra patria ${ }^{4}$.

Tras el fallecimiento de su amigo Haenseler, en 1841, heredó Prolongo su completo herbario. Creemos que la primera publicación de éste fue la de 1842 titulada "Estudio mineralógico y geológico de la provincia de Málaga, y determinación de la sulfuraria que vive en las aguas de Carratraca"'. Continúa en la década siguiente con el catálogo botánico "Plantas de Málaga y su término", que fue incluido en la Topografía médica de la ciudad de Málaga publicada por el facultativo militar Vicente Martínez Montes $(1852)^{6}$. Al año siguiente la Sociedad Económica de Amigos del País de Málaga premia a Prolongo, y edita su Memoria sobre la enfermedad de la vid..., precedida del acuerdo de la Sociedad, y del informe de la Comisión nombrada para examinarla (1853); donde analiza la enfermedad causada por Oidium tuckeri. El texto fue ampliamente comentado en varios periódicos de la época, como el Boletín Oficial del Ministerio de Fomento (1854), o la Revista de los Progresos de las Ciencias Exactas, Físicas y Naturales (1854); la Real Academia de Ciencias de Madrid le concedió una 'mención honorífica' el mismo año ${ }^{7}$.

Entre su obra impresa J. L. Carrillo ha destacado: "Historia de los copos de azufre que salen mezclados con las aguas del manantial de Carratraca (Sulfuraria Carratraquensis)" publicado en 1874, trabajo en el que abordó aspectos de embriogenia vegetal, y "Monstruosidades del género Citrus y los caracteres de ese fruto", editado en 1875 , en el que se puso de manifiesto el interés del estudio de las anomalías como vía para conocer la organografía y la fisiología vegetal ${ }^{8}$. Otros de sus textos originales fueron "Irregularidades de los frutos de las Auranciaceas" (éste fue motivo de una honrosa distinción por la Sociedad Botánica de Brandeburgo, en 16/1/1876), "Mapa botánico de la provincia de Málaga" (que se supone perdido), "Sinonimia de la flora española Chloris Malacitana, o sea breve exposición de la verdura de esta provincia"; "Sobre los insectos que atacan cada planta en diversas épocas del año" (un estudio que quedó en proyecto); "Excursión botánica y geológica" (un viaje por la sierra de Mijas) y "Estudio sobre vinos". Varios de estos trabajos no llegaron a publicarse y otros se consideraban ya perdidos en los años treinta del siglo XX. [Aportamos más datos biográficos de Prolongo en el artículo de Pérez-Rubín, presente volumen].

\section{El artículo de Pablo Prolongo en La Clínica de Málaga (1880)}

En el primer número de esa revista profesional, aparecido en enero de $1880^{10}$, vio la luz un curioso artículo del boticario Pablo Prolongo sobre el jarabe de abietina ("precioso medicamento"), que no consta en las reseñas que de su producción científica (publicaciones y manuscritos) hemos consultado. Por este motivo y su interés lo transcribimos a continuación. Enél recuerda el autor sus ya lejanas herborizaciones de 1837, acompañado de sus colegas Boissier y Haenseler. Intuye nuestro boticario que su preparado curativo podría ser beneficioso para la curación de muy diferentes enfermedades, aunque particularmente indicado para las afecciones respiratorias, e indica las dosis para 
el tratamiento de adultos, jóvenes y niños, bajo supervisión médica.

El principio activo de su jarabe era la abietina descrita por Caillot, ya conocida al menos en $1845^{11}$, aunque cuatro décadas después eran únicamente tres especies de Abies las que se consideraban más útiles en medicina $^{12}$.

"Jarabe Pectoral de Abietina preparado por el Dr. D. Pablo Prolongo y García, con la savia del Abies pinsapo de Mr. Boissier."

El árbol de cuya savia se obtiene el principio activo para la confección de este precioso medicamento, es una planta propia de nuestro suelo, y que por no ser reconocido en la época de su florescencia, fue clasificado por Lamark, como una especie diferente denominada Pinus maritima.

El célebre naturalista D. Simón de Rojas Clemente y el eminente sabio D. Félix Haensseler, alemán naturalizado en España, farmacéutico muy versado en ciencias naturales y corresponsal de varias sociedades científicas, en 1808 fueron de opinión, que el árbol designado por Lamark con el nombre de Pinus maritima, era otra especie, si bien no hallándolo en flor no pudieron clasificarlo.

En 1837 Mr. Boissier, D. Félix Haenseler y yo, herborizando juntos, lo encontramos en fruto, y tuve el honor de ser encargado por mis sabios compañeros de excursión, para describir los órganos florales de dicha planta, en la primavera próxima, con cuyos datos obtenidos por mí quedó clasificado Abies pinsapo (de Boissier).

Los productos óleos-resinosos y las resinas de las diversas especie del género Abies, son ligeramente amarillos, de un aroma y sabor agradable, balsámicos muy estimados y de elevado precio; contiene, según el análisis de Mr. Caillot, en varias proporciones una sustancia cristalizable a la que denomina abietina y un ácido que él llama ácido abiético ${ }^{13}$.
El uso de los varios productos, obtenidos de las diversas partes de los árboles del género Abies, han sido siempre usados y recomendados en todos los tratados de materia médica, bajo la forma de cocimiento, tisana, extracto, tintura alcohólica y jarabe; el médico obtiene siempre resultados eficaces en la práctica y tratamiento de muchas enfermedades y especialmente en la afecciones catarrales, tisis incipiente, congestiones pulmonales [sic], diversos padecimientos de las vías urinarias, antiescorbúticos y diuréticos; siendo muy preconizado por varios autores como antigotosos vulnerarios y anti-reumáticos.

El Jarabe de Abietina preparado con la savia del Abies pinsapo (Boissier), además del gusto agradable y olor aromático, tiene la gran ventaja de ser bien soportado por el estómago, cuyo órgano esperimenta [sic] modificaciones muy favorables con el uso de este precioso medicamento. La gran ventaja de poder obtenerlo reciente, sin que halla sufrido resinificación [sic] alguna la savia de esta planta española y de nuestra propia provincia, garantiza la bondad del específico.

\section{Manera de administrar el Jarabe de Abietina}

El Jarabe de Abietina debe usarse tomando tres cucharadas de las de sopas al día, una por la mañana, otra a medio día antes de comer y otra por la noche para los adultos. Para los niños debe seguirse el mismo método, solo usando cucharaditas pequeñas desde el tamaño de las que se usan para el café hasta las de mediano tamaño según la edad del joven.

Cuando el alivio sea notable la cantidad del jarabe puede aumentarse o disminuirse según el médico lo crea conveniente".

\section{NOTAS}

${ }^{1}$ A. González Bueno. Acta Botanica Malacitana, 35: 5-21. 2010

${ }^{2}$ B. Cabezudo et al. Acta Botanica Malacitana, 35: 
195-204. 2010.

${ }^{3}$ E. Boissier. Annales des Sciences Naturelles, 9: 167-172. 1838.

${ }^{4} \mathrm{R}$. Casares López. Anales de la Academia Nacional de Farmacia, 4: 49-57. 1932.

${ }^{5}$ Referencia extraída de la bibliografía del artículo de A. Rotureau sobre "Las Aguas Minerales de Carratraca o Ardales", incluido en el Dictionnaire encyclopédique des sciences médicales (Tomo XII: 690-693). París, 1874. Muy probablemente Prolongo le facilitó la exhaustiva relación de 14 referencias españolas que incluye el francés como fuentes (del período 1736-1860).

${ }^{6} \mathrm{~V}$. Martínez Montes. Topografía médica de la ciudad de Málaga. Málaga, 1852. En la página 64 se concreta la autoría: "El Catálogo de Plantas, así espontáneas como cultivadas, que a continuación presentamos, que debemos a la amistosa deferencia de este entendido botánico [Prolongo]". Dicho amplio "Catálogo..." (páginas 73-127) abarca más de 800 especies, en él se aportan los nombres linneanos de las especies y presta atención a las plantas medicinales.

${ }^{7} \mathrm{La}$ Revista de los Progresos de las Ciencias Exactas, Físicas y Naturales, editada por la Real Academia de Ciencias de Madrid, publicó en su volumen correspondiente a 1854 (Tomo IV: 250), una reseña del contenido y de su mérito: “Además de presentar en ella un bosquejo de las condiciones generales de salud y enfermedad de las plantas, contiene muchas observaciones sobre el origen, circunstancias, causas patológicas del desarrollo de la enfermedad de la vid y elección de remedios para combatirla. La Real Academia de Ciencias acordó en sesión del 27 de febrero último [1854], que estando ya impresa la citada Memoria, se manifestase al autor que la había recibido con aprecio, y que se hiciese mención honorífica de ella en sus actas".

${ }^{8}$ J. L. Carrillo. En: M. Alcobendas (ed.). Málaga. Personajes en su historia: 357-358. Málaga. 1986.

${ }^{9} \mathrm{R}$. Casares López (Op. cit. nota 4. pág. 56) comenta que Prolongo mantuvo correspondencia científica con Renter, Kunk, Weh [sic], Rojas Clemente y el propio Boissier.

${ }^{10}$ La Clínica de Málaga (Revista de Medicina y Cirugía Prácticas). 1 (1): 23-24. Málaga. 1880. La revista fue el órgano oficial del Colegio Médico de Málaga; al menos este primer número, correspondiente a enero de 1880, fue impreso en 'Establecimiento tipográfico El Mediodía'. Como redactores responsables de la revista figuran: Aurelio Abela de la Torre, Antonio Linares Enríquez, Antonio Alonso de Navas y Sebastián Pérez Souvirón.

${ }^{11} \mathrm{Ch}$. Gerhardt. Précis de chimie organique (Tomo
II: 112). Paris, 1845. La abietina o resina-gamma de Berzélius, procedente de Pinus picea y Abies.

${ }^{12} \mathrm{E}$. Ferrand. Aide-Mémoire de Pharmacie. Vade-mecum du Pharmacien. A l'Officine et au Laboratoire (Tomo 1: 693-694). 1883. Figuran las tres especies: Abies balsamea, A. excelsa y A. pectinata. Se concreta que esta última contenía, aparte de la abietina ("resina neutra, cristalizable") una resina ácida (ácido abiético).

${ }^{13}$ Véanse las dos notas anteriores.

Dirección de los autores: Centro Oceanográfico de Málaga. Instituto Español de Oceanografía. Muelle Pesquero s/n. Apdo. 285. 29.640 Fuengirola (Málaga).Dirección de contacto: jprubin@ma.ieo.es. 